Published in final edited form as:

Nature. 2011 February 17; 470(7334): 359-365. doi:10.1038/nature09787.

\title{
Telomere dysfunction induces metabolic and mitochondrial compromise
}

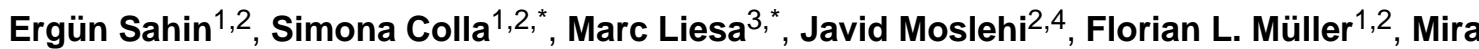
Guo $^{5}$, Marcus Cooper ${ }^{6}$, Darrell Kotton ${ }^{3}$, Attila J. Fabian ${ }^{7}$, Carl Walkey ${ }^{8}$, Richard S. Maser $^{1,2}$, Giovanni Tonon ${ }^{1,2}$, Friedrich Foerster ${ }^{1,2}$, Robert Xiong ${ }^{1}$, Y. Alan Wang ${ }^{1}$, Sachet A. Shukla ${ }^{1}$, Mariela Jaskelioff ${ }^{1,2}$, Eric S. Martin ${ }^{1,2}$, Timothy P. Heffernan ${ }^{1}$, Alexei Protopopov $^{1}$, Elena Ivanova ${ }^{1}$, John E. Mahoney ${ }^{1}$, Maria Kost-Alimova ${ }^{1}$, Samuel R. Perry ${ }^{1}$, Roderick Bronson ${ }^{9}$, Ronglih Liao ${ }^{4}$, Richard Mulligan ${ }^{7}$, Orian S. Shirihai ${ }^{3}$, Lynda Chin ${ }^{1,2}$, and Ronald A. DePinho ${ }^{1,2,4,7}$

${ }^{1}$ Belfer Institute for Applied Cancer Science, Dana-Farber Cancer Institute, Boston, Massachusetts 02115, USA

${ }^{2}$ Department of Medical Oncology, Dana-Farber Cancer Institute, Harvard Medical School, Boston, Massachusetts 02115, USA

${ }^{3}$ Department of Medicine, Boston University School of Medicine, Massachusetts 02118, USA

${ }^{4}$ Department of Medicine, Brigham and Women's Hospital, Harvard Medical School, Boston, Massachusetts 02115, USA

${ }^{5}$ School of Engineering and Applied Sciences, Harvard University, Cambridge, Massachusetts 02138, USA

${ }^{6}$ Division of Cardiovascular Medicine, University of Massachusetts, Worcester, Massachusetts 01605, USA

${ }^{7}$ Department of Genetics, Harvard Medical School, Boston, Massachusetts 02115, USA

${ }^{8}$ St Vincent's Institute and Department of Medicine, St Vincent's Hospital, University of Melbourne, Victoria 3065, Australia

${ }^{9}$ Rodent Histopathology Laboratory, Harvard Medical School, Boston, Massachusetts 02115, USA

\section{Abstract}

(C)2011 Macmillan Publishers Limited. All rights reserved

Correspondence and requests for materials should be addressed to R.A.D. (ron_depinho@dfci.harvard.edu).

These authors contributed equally to this work.

Supplementary Information is linked to the online version of the paper at www.nature.com/nature.

Author Contributions E.S. performed all experiments and contributed to echocardiographies (J.M., R.L.), mitochondrial respiration studies (M.L., O.S.S., F.L.M., M.C.). S.C. was involved in microarray analysis and most of other experiments. G.T., R.X. and S.A.S. contributed to microarray analysis. D.K., A.J.F., C.W., M.J. and R.M. advised and helped with transplantation experiments. A.P., E.I., J.E.M., M.K.-A., S.R.P. helped with immunohistochemistry and peptide-nuclei-acid-probes-based fluorescence in-situ hybridization (PNA-FISH) studies, R.S.M. and F.F. provided MEFs and helped with MEF studies. R.B. assessed histological slides. E.S.M., T.P.H. and M.G. helped with ChIP experiments. M.G. helped with qPCR experiments. L.C. supervised bioinformatic analysis. L.C. and Y.A.W. helped with writing and contributed intellectually. E.S. and R.A.D. conceived the ideas, designed experiments and wrote the manuscript.

Author Information Reprints and permissions information is available at www.nature.com/reprints. The authors declare no competing financial interests. Readers are welcome to comment on the online version of this article at www.nature.com/nature. 
Telomere dysfunction activates p53-mediated cellular growth arrest, senescence and apoptosis to drive progressive atrophy and functional decline in high-turnover tissues. The broader adverse impact of telomere dysfunction across many tissues including more quiescent systems prompted transcriptomic network analyses to identify common mechanisms operative in haematopoietic stem cells, heart and liver. These unbiased studies revealed profound repression of peroxisome proliferator-activated receptor gamma, coactivator 1 alpha and beta ( $P G C$ - $1 a$ and $P G C-1 \beta$, also known as Ppargcla and Ppargc1b, respectively) and the downstream network in mice null for either telomerase reverse transcriptase (Tert) or telomerase RNA component (Terc) genes. Consistent with PGCs as master regulators of mitochondrial physiology and metabolism, telomere dysfunction is associated with impaired mitochondrial biogenesis and function, decreased gluconeogenesis, cardiomyopathy, and increased reactive oxygen species. In the setting of telomere dysfunction, enforced Tert or PGC-1a expression or germline deletion of $p 53$ (also known as Trp53) substantially restores PGC network expression, mitochondrial respiration, cardiac function and gluconeogenesis. We demonstrate that telomere dysfunction activates p53 which in turn binds and represses $P G C$ - $1 a$ and $P G C$ - $1 \beta$ promoters, thereby forging a direct link between telomere and mitochondrial biology. We propose that this telomere-p53-PGC axis contributes to organ and metabolic failure and to diminishing organismal fitness in the setting of telomere dysfunction.

\begin{abstract}
Multiple biological processes driven by diverse molecular factors conspire progressively to diminish organ function with advancing age. Molecular and cellular analyses of age-related conditions such as muscle atrophy, diabetes and cardiomyopathy implicate diminished mitochondrial function and telomere dysfunction in driving pathogenesis ${ }^{1-3}$. Aged tissues show accumulation of mitochondrial DNA (mtDNA) mutations causing respiratory chain deficiency and increased reactive oxygen species (ROS) ${ }^{2,4}$, which may underlie declining mitochondrial energy production and progressive loss of vigour in the aged ${ }^{5}$. The importance of mitochondrial integrity for healthy ageing is reinforced by premature ageing in mitochondrial polymerase (PolgA) mutant mice, which sustain increased mtDNA mutations ${ }^{6}$. Whereas the instigating processes driving age-associated mitochondrial decline are not known, in the context of this study, it is notable that the activity of the master mitochondrial regulators PGC-1 $\alpha$ and PGC-1 $\beta$ decreases across ageing tissues ${ }^{7,8}$. The relevance of PGCs to age-related pathologies may stem from their regulation of mitochondrial biogenesis and control of metabolic processes (gluconeogenesis, fatty acid metabolism and $\beta$-oxidation), processes that relate to increased insulin resistance and diabetes in the aged ${ }^{9}$.
\end{abstract}

Increasing evidence also implicated telomeres in the pathogenesis of age-related disorders ${ }^{10}$. Telomeres are nucleoprotein complexes at chromosome ends that function to preserve chromosomal integrity and quell p53-dependent DNA damage and DNA repair activity at these free ends ${ }^{11}$. In the absence of telomerase, continued cell division results in telomere shortening, loss of 'capping' function, and p53 activation ${ }^{12-15}$. The prevailing view of how uncapped telomeres compromise organ function posits that p53 mediates cellular checkpoints of growth arrest, senescence and apoptosis in stem/progenitor cells ${ }^{10,16}$. Correspondingly, p53 deficiency ameliorates these phenotypes, particularly in organs with high proliferative demands supported by resident stem cells ${ }^{15}$. The relevance of p53 in ageing is evidenced by stem cell depletion and premature ageing in mice engineered with hyper-active p53 alleles ${ }^{17,18}$.

Whereas stem/progenitor cell failure due to p53-mediated cellular checkpoints may underlie compromise of highly proliferative organs, this mechanism seems inadequate to explain the profound physiological decline in more quiescent tissues, for example, heart (cardiomyopathy) and liver (reduced detoxification capacity, glucose intolerance). These 
pathologies indicate that telomere dysfunction elicits a degenerative state via additional mechanisms beyond the classical senescence and apoptosis checkpoints.

\section{Telomere dysfunction represses PGC network and mitochondrial biogenesis}

$\mathrm{Tert}^{-/}$mice were intercrossed to produce successive generations of telomerase deficient mice with decreasing telomere reserves. The $\mathrm{Tert}^{+/+}$controls, first generation (G1) $\mathrm{Tert}^{-/-}$ (telomerase deficient with largely intact telomeres), and fourth generation (G4) $\mathrm{Tert}^{-/}$ (telomerase deficient with severe telomere dysfunction) are designated hereafter as 'WT', 'G1' and 'G4' mice. In this model, progressive telomere shortening is associated with increasing atrophy and functional decline across proliferative organs (intestinal, haematopoietic) and equally profound impairment in relatively quiescent organs (liver and heart) that do not exhibit significant levels of apoptosis (Supplementary Fig. 1; not shown). Body composition analysis showed body weight reduction and striking decrease in fat mass with preserved lean mass, yet no differences in spontaneous activity or food intake were apparent, while running endurance capacity was significantly reduced in G4 mice (not shown). G4 mice also showed decreased blood triglyceride and cholesterol levels and increased free fatty acids (not shown). Thus, telomere dysfunction in the $\mathrm{Tert}^{-/}$model engenders a premature ageing condition with severe anatomic and functional compromise across diverse organ systems.

To illuminate mechanisms underlying the broad impact of telomeres, we profiled 'proliferative' (CD34low/- c-Kit+ Sca-1+ Lin-, hereafter haematopoietic stem cells, 'HSCs') and 'quiescent' (liver and heart) tissues from 12-16-week-old WT, G1 and G4 mice. Telomere dysfunction transcriptomes showed strong enrichment for networks regulated by PGC- $1 \alpha$ and PGC- $1 \beta$ including oxidative phosphorylation (OXPHOS), mitochondrial function, oxidative stress and gluconeogenesis (Fig. 1; Supplementary Tables 1-3). G1 transcriptomes showed modest downward trends consistent with less telomere dysfunction (Fig. 1). The most prominent network perturbations centred on repressed OXPHOS genes, among which are many PGC targets (Supplementary Fig. 2;

Supplementary Tables 1-3), and on repression of key genes governing oxidative defence (Sod1, glutathione peroxidase), gluconeogenesis (Pepck, Glc-6-P), fatty acid and cholesterol synthesis (Fasn, Dgat, Gpat Hmgcs1, Hmgcr) and $\beta$-oxidation (Acadm, Acadl and Cpt1c, respectively). Quantitative reverse transcriptase polymerase chain reaction analysis (RTqPCR) and western blot analysis of G4 tissues confirmed decreased PGC-1 $\alpha$ and PGC- $1 \beta$ expression (Fig. 1) and their critical targets such as NRF-1, ERRa (also known as ESRRA), PPARa and TFAM (Fig. 1; Supplementary Fig. 3). G1 tissues showed marginal reductions in PGC-1a, PGC-1 $\beta$ and their target genes (Fig. 1). Thus, telomere dysfunction represses PGC- $1 \alpha / \beta$ and their downstream gene network, which controls many aspects of mitochondrial biology and cellular metabolism ${ }^{9}$.

\section{Telomere dysfunction decreases mitochondrial mass and energy production}

The above profiles prompted assessment of whether telomere dysfunction has an impact on mitochondrial biology. We documented reduced mitochondrial DNA content (Fig. 2a) and reduced mitochondrial density in G1 and G4 heart and liver (Supplementary Fig. 4a and b). Functionally, telomere dysfunction correlated with a marked decline in complex I and IV activity when assayed with either whole tissue lysates or isolated mitochondria from G1 and G4 heart and liver tissues (Supplementary Fig. 4c). The functional compromise of mitochondria was further substantiated by respiration studies of isolated liver and heart 
mitochondria from WT and G4 mice using the Seahorse XF24 (see Supplementary data online). State III (ADP-stimulated) respiration assay revealed a significant reduction in $\mathrm{O}_{2}$ consumption in G4 heart and liver mitochondria (Fig. 2b; Supplementary Fig. 5a). Furthermore, respiration uncoupled from ATP synthesis (induced by 2,4-dinitrophenol, DNP) was also reduced in G4 liver and heart mitochondria (31\% and $25 \%$, respectively) (Fig. 2b). Additionally, complex II- and complex IV-dependent respiration was also decreased in G4 mitochondria (Supplementary Fig. 5a). Clark electrode-based studies in isolated liver mitochondria showed similar compromise in respiration (Supplementary Fig. $5 b)$. Together, these results demonstrate defective electron transport chain activity in both G4 liver and heart mitochondria. In this regard, G4 heart mitochondria displayed significantly decreased ATP synthesis rates (Fig. 2c). This impaired mitochondrial ATP synthesis is associated with decreased total ATP content in G4 hearts and livers (Fig. 2d).

In addition, decreased expression of ROS detoxifying enzymes including known PGC-1a targets was observed in G4 HSCs, liver and heart, which aligned with increased ROS levels by FACS in HSCs and increased carbonylated proteins in liver homogenates (Supplementary Fig. 6a-c). Further, complex I and complex II dependent $\mathrm{H}_{2} \mathrm{O}_{2}$ production rates were significantly increased in G4 mitochondria (Supplementary Fig. 6d). However, G1 or G4 mice treated from birth with $N$-acetylcysteine, a ROS scavenger, did not rescue defective HSC repopulation in competitive transplantations (Supplementary Fig. 6e), indicating that increased ROS is not mechanistically central to HSC failure.

Taken together, these collective data establish that telomere-dysfunction-induced repression of the PGC network is associated with mitochondrial dysfunction as evidenced by compromised OXPHOS and respiration, decreased ATP generation capacity, and increased oxidative stress. Importantly, given evidence of non-telomere-related functions of Tert ${ }^{19,20}$, we also demonstrated that, with onset of telomere dysfunction, $\mathrm{Terc}^{-/-}$mice (normal Tert expression) experience degenerative phenotypes indistinguishable of those in the $\mathrm{Tert}^{-1-}$ model $^{21,22}$. Specifically, G2 $\mathrm{Terc}^{-/-}$liver and hearts (exhibiting comparable telomere dysfunction as G4 $\mathrm{Tert}^{-/-}$mice) exhibited the same canonical pathway alterations (Supplementary Fig. 7a; Supplementary Tables 4 and 5) as well as reductions in PGC and targets, mitochondrial DNA content, complex I and IV activity, and ATP levels (Supplementary Fig. 7b-e). The indistinguishable mitochondrial and energy profiles of Tert and Terc models indicate that telomere dysfunction per se is the principal factor driving these phenotypes.

\section{Telomere dysfunction induces pathophysiologies classical for impaired PGC function}

Although these findings link dysfunctional telomeres to diminished PGC co-activator expression, we sought additional physiological evidence of such correlations in vivo. Previous work has established that repression of either or both co-activators (PGC-1a or PGC-1 $\beta$ ) decreases mitochondrial biogenesis and function, elicits cardiomyopathy and impairs de novo glucose production ${ }^{23-25}$. G4 Tert $^{-/}$mice exhibited these classical signs of impaired PGC function, including an age-progressive dilated cardiomyopathy with left ventricular wall thinning, increased left ventricular diameter, and reduced fractional shortening by 15 months of age (Fig. 3a), as reported previously in $\mathrm{Terc}^{-/}$mice $^{26}$. G4 mice also exhibited an inability to maintain plasma glucose levels under fasting conditions, experiencing a marked $30 \%$ drop at $12 \mathrm{~h}$ of fasting, yet maintained normal plasma glucose levels under fed conditions (Fig. 3b). This defect in gluconeogenesis was also evident in cultured hepatocytes from G4 mice (not shown), indicating a cell intrinsic defect. Finally, prompted by studies linking impaired mitochondrial function to defective HSC maintenance ${ }^{27-29}$, we showed that either enriched HSC or whole bone marrow derived from 
G4 mice show decreased capacity to reconstitute lethally irradiated recipients in competitive transplant experiments (Fig. 3c, not shown).

Additionally, to genetically strengthen the telomere-PGC-mitochondrial link in vivo, we reintroduced Tert in G4 livers via adenoviral delivery and monitored gene expression and gluconeogenic output under fasting conditions. Five days following Ad-Tert injection, partial rescue of PGC-1a/ $\beta$, Glc-6-P and Pepck expression (Supplementary Fig. 8a) was evident, accompanied by a $30 \%$ increase in glucose levels relative to Ad-GFP controls; AdTert had no effect on gluconeogenesis in WT mice (Fig. 3d). We excluded multi-system causes affecting glucose control by documenting similar Ad-Tert responses in cultured G4 hepatocytes which show an $80 \%$ increase in glucose output relative to Ad-GFP controls (Supplementary Fig. 8b). Furthermore, this PGC-mitochondrial link is supported by AdPGC-1a-induced rescue of gluconeogenesis in vivo and in vitro in G4 hepatocytes (Fig. 3e; Supplementary Fig. 8c). This Ad-PGC-1a impact was markedly more pronounced in G4 mice than in WT mice or hepatocytes. Finally, to test whether PGC overexpression can also reverse the mitochondrial respiration defect in telomere dysfunctional mice, we measured respiration in isolated liver mitochondria after adenoviral PGC delivery in vivo. One week after transduction, isolated liver mitochondria transduced with PGC-1a showed an increase in state III (3.1-fold) and state IV (3.2-fold) respiration compared to GFP-transduced controls (Fig. 3f). WT mitochondria transduced with Ad-PGC showed increased respiration, albeit at lower levels (state III 1.8-fold; state IV 2.1-fold increase; not shown).

\section{p53 directly represses PGC-1 $\alpha$ and PGC-1 $\beta$}

p53 deficiency markedly attenuates the degenerative phenotypes associated with telomere dysfunction, whereas constitutively activated p53 accelerates ageing phenotypes ${ }^{15-18}$. This role in ageing and p53's activation in telomere dysfunctional tissues of $\mathrm{Terc}^{-/-}$mice and Tert $^{-/-}$mice (Supplementary Fig. 9a-d) prompted assessment of direct links between p53 activation and PGC-1a/ $\beta$ regulation on multiple levels. First, relative to $22 \mathrm{Terc}^{-/-} p 53^{+/+}$ littermate controls, G2 $\mathrm{Terc}^{-/-} p 53^{-/-}$liver and hearts showed substantial or complete restoration of PGC-1 $\alpha$ and PGC-1 $\beta$ expression (Fig. 4a) and downstream targets ERRa, PPAR $\gamma$ and TFAM (not shown). Second, mitochondrial DNA copy number is significantly restored in G2 $\mathrm{Terc}^{-/} p 53^{-/-}$livers and hearts relative to age- and gender-matched G2 $\mathrm{Terc}^{-/} p 53^{+/+}$controls (Fig. 4b). Third, G2 $\mathrm{Terc}^{-/} p 53^{-/-}$MEFs harbouring a 4hydroxytamoxifen (OHT)-induced p53-oestrogen receptor fusion construct (p53-ER) demonstrated potent OHT-dependent repression of PGC-1 $\alpha$, PGC- $1 \beta$ and downstream targets within $4 \mathrm{~h}$ of OHT treatment (Supplementary Fig. 10a; data not shown). Fourth, after

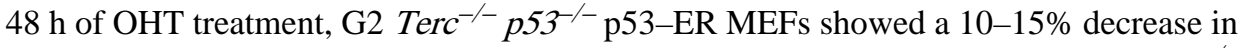
mitochondrial mass compared with no significant change in the OHT-treated G2 $\mathrm{Terc}^{-/}$ $p 53^{-/}$controls (Supplementary Fig. 10b). Fifth, in silico promoter analysis (p53MH program) identified potential p53 binding elements in PGC- $1 a$ and PGC-1 $\beta$ promoters. These promoter regions were cloned into the PGL4 reporter and transfected into G2 $\mathrm{Terc}^{-1-}$ $p 53^{+/+}$MEFs which showed fivefold repression of PGC-1a-PGL4 and PGC-1 $\beta-P G L 4$ reporter activity relative to G2 $\mathrm{Terc}^{-/-} \mathrm{p} 53^{-/-}$MEFs (Fig. 4c). Positive (p21 responsive reporter, PG-13) and negative (empty vector PGL4; mutated p21 reporter 'MG') control reporters showed the expected reporter activity (Fig. 4c). Repressed reporter activity was also observed following transfection of a pCDNA-p53 expression vector into G2 $\mathrm{Terc}^{-/-}$ $p 53^{-/}$MEFs harbouring PGL-4-PGC-1 $a$ and PGL4-PGC-1 $\beta$ reporters (not shown). Finally, anti-p53 chromatin-immunoprecipitation assays in $\mathrm{G} 2 \mathrm{Terc}^{-/-} \mathrm{p} 53^{+/+}$, but not $\mathrm{G} 2$ $\mathrm{Terc}^{-/-} \mathrm{p} 53^{-/-}$, MEFs demonstrated physical binding between p53 and (1) PGC-1a fragments containing p53 binding elements at -564 and -954 , but not -2317 , and (2) PGC1 $\beta$ fragments containing p53 binding elements at -1616 and -1030 (Fig. 4d). 
To test whether the herein and elsewhere documented ${ }^{30}$ negative impact of p53 on mitochondrial function is context-specific, we overexpressed p53 at similar levels in $p 53^{-/-}$ and G2 $p 53^{-/-}$MEFs by adenoviral delivery (Supplementary Fig. 11a). Increasing dosage of p53 in either $p 53^{-/}$or G2 $p 53^{-/}$MEFs showed repressed PGC- $1 a$ and PGC-1 $\beta$ levels and increasing occupancy of $\mathrm{p} 53$ binding elements in PGC- $1 \alpha$ and PGC- $1 \beta$ promoters (Supplementary Fig. 11b, c). Furthermore, WT MEF or heart tissues of mice treated with the DNA damage agent doxorubicin showed increased p53 levels after $24 \mathrm{~h}$ and coincident binding of p53 to PGC-1a promoter and repression of PGC-1a levels as documented before $^{31}$ (not shown). This repression was significantly rescued by p53 deficiency in MEFs and in hearts treated with doxorubicin (not shown). Thus, increasing p53 levels can lead to PGC repression in these genetic contexts.

The functional relevance of $\mathrm{p} 53$-mediated repression of PGC-1a/ $\beta$ was examined by assessment of the impact of p53 status on liver and cardiac function. In liver, we observed higher gluconeogenic capacity upon fasting in G4 $p 53^{-/}$mice (Fig. 5a, top) and isolated hepatocytes relative to that of G4 $p 53^{+/+}$controls (Fig. 5a). In heart, a protocol of low-dose doxorubicin-induced cardiomyopathy showed $30 \%$ improvement of fractional shortening in $\mathrm{G} 4 p 53^{-/-}$mice relative to that of G4 $p 53^{+/+}$controls (Fig. 5b). In mice with intact telomeres, p53 status had minimal impact on liver gluconeogenesis or cardiac function. In summary, combined biochemical, genetic and physiological results indicate that (1) activated p53 directly represses PGC-1 $\alpha$ and PGC-1 $\beta$, (2) p 53 deficiency alleviates transcriptional repression and associated decline in mitochondrial DNA content in tissues and cells with telomere dysfunction, and (3) p53 deficiency partially rescues telomere dysfunction-induced heart and liver pathophysiologies which have been classically associated with PGC deficiencies and mitochondrial dysfunction.

\section{Discussion}

Telomere dysfunction has been linked to many aspects of the ageing process. Genetic model systems have established the essentiality of preserved telomere function in the regenerative maintenance of highly proliferative organs as well as in the preservation of physiological functions of more quiescent organs such as heart, brain and liver. Although classical p53directed checkpoints of proliferative arrest and apoptosis have provided a strong basis for atrophy and functional impairment of high turnover tissues, the mechanistic basis for comparably severe compromise of less proliferative tissues has been an enigma. Here, combined transcriptomic, molecular, genetic and functional analyses on cellular and organismal levels established a direct molecular link between telomere dysfunction and repression of PGC-dependent processes of mitochondrial biogenesis/function, gluconeogenesis and oxidative defence. This combined repression of PGC-1 $a$ and PGC-1 $\beta$ and associated decline in mitochondrial biogenesis and function as well as metabolic changes aligns well with the phenotypes elicited by combined PGC- $1 \alpha$ and PGC- $1 \beta$ knockdown in vitro or knockout in vivo ${ }^{25,32}$. We propose that telomere dysfunction-induced repression of the PGC network and consequent mitochondriopathy and metabolic changes, along with telomere-induced apoptotic and proliferative checkpoints, contributes to functional multi-system decline in the setting of telomere dysfunction.

Given the diverse roles of PGC in multiple biological processes, the relative contribution of each of the dysregulated PGC arms to functional decline in the setting of telomere dysfunction may differ in various tissue types. For example, the PGC-directed mitochondrial dysfunction might be particularly critical in heart, whereas changes in metabolic gene expression may predominate in liver. Of potential clinical interest is the link of PGC to insulin resistance and diabetes in ageing, both of which are correlated with short telomeres ${ }^{33,34}$. These mitochondrial and metabolic aspects of telomere-driven 
pathophysiology are not mutually exclusive with the well documented 'DNA damage' checkpoints associated with telomere dysfunction. These DNA damage checkpoints manifest primarily as cellular senescence and/or apoptosis which contribute to depletion and compromise of tissue stem cell reserves, particularly in highly proliferative organs such as intestine, skin and blood ${ }^{13,35,36}$. Although the relative contributions of these checkpoints versus metabolic/mitochondrial dysfunction in a given degenerative phenotype will require further study, the latter mechanism may explain the profound physiological impairment observed in the more static, post-mitotic tissues such as heart (cardiomyopathy) and liver (impaired gluconeogenesis), leading to feeble energy stress responses and overall frailty due to a fundamental inability to produce adequate cellular ATP. As with other mitochondriopathies, this mitochondrial defect in the setting of telomere dysfunction becomes increasingly apparent under conditions of physiological stress or advanced age ${ }^{2}$. Whereas the instigator of decline in advanced age is not known, our findings raise the possibility that the well-known phenomenon of accelerating physiological decline in the aged could stem from a primary decline in PGC/mitochondrial function which in turn would lead to increased ROS, resulting from the diminished OXPHOS/complex activity and decreased expression of PGC-regulated oxidative defence genes. These increased ROS levels would set in motion a detrimental cycle of genotoxic damage with rapid erosion and damage of G-rich telomeres, sustained p53 activation, further repression of PGC, progressive mitochondrial decline, and so on ${ }^{37,38}$. Under such circumstances, pharmacological correction of increased ROS would not be expected to substantially correct the primary mitochondrial defect and associated degenerative phenotypes, although such interventions may slow the rate of decline. Finally, beyond ROS accumulation, yeast genetic studies have shown that mitochondrial dysfunction results in a decline in iron-sulphur cluster biogenesis ${ }^{39}$ which can cause nuclear genomic instability and thus would be expected to drive further decline in mitochondrial function via genotoxic activation of p53 and associated repression of PGC. It is also worth noting that p53-mediated repression of PGC is highly context dependent - shown here to occur in the setting of telomere dysfunction with increased p53 levels and activity — and that p53 can exert varied effects on mitochondrial biogenesis and function in other tissue and physiological settings depending on levels and kinetics of p53 activation, among other factors ${ }^{30,40}$.

Mitochondria use oxidative phosphorylation to convert dietary intake into ATP, and in the process, generate ROS which can damage mitochondrial DNA, impair respiratory chain function, and cause nuclear DNA damage and cellular checkpoint activation. Given the central importance of mitochondria, one might anticipate that any genetic manipulation resulting in significantly decreased mitochondrial biogenesis/activity could accelerate the ageing process and cause age-related disorders such as diabetes, heart disease and neurological decline. From this perspective, it is worth noting that there are many molecules with important roles in organismal ageing or age-related disorders which interact directly with components of the telomere-mitochondrial axis. In particular, the increased lifespan associated with caloric restriction in model organisms is accompanied by increased mitochondrial density and respiration. Caloric restriction is associated with increased SIRT1 activity, which stabilizes PGC-1a in turn increasing mitochondrial biogenesis and function $^{41}$. Moreover, the beneficial impact of SIRT1 may also stem from its deacetylation and inactivation of p53, which may attenuate checkpoint responses and de-repress PGC-1a expression; conversely, SIRT1 knockout mice show widespread p53 activation and shortened life expectancy ${ }^{42,43}$.

In summary, multiple levels of evidence establish telomere dysfunction-induced p53 represses PGC-1 $a$ and PGC-1 $\beta$, thereby linking telomeres to mitochondrial biology, oxidative defence, and metabolism. As illustrated in Fig. 5c, this telomere-p53-PGC 
pathway expands our understanding of how telomere dysfunction may compromise organ function and contribute to age-related disorders.

\section{METHODS SUMMARY}

$\mathrm{Terc}^{-/-}$and $\mathrm{Tert}^{-/}, \mathrm{p53}^{-/-}$mice have been described previously ${ }^{15,44}$. Microarray analysis of HSCs, heart and liver tissues from WT, G1 and G4 $\mathrm{Tert}^{-1-}$ and G2 $\mathrm{Terc}^{-/-}$mice was performed using either SAM (liver and heart) or fold change differences (HSCs) followed by Ingenuity pathway analysis (IPA). Quantitative RT-qPCR was analysed by $\Delta \Delta C \mathrm{t}$ method. qPCR-based mitochondrial quantification was performed with two different primer sets for genomic and mitochondrial loci. Shock-frozen heart and liver tissues were used for ATP determination by HPLC. Mitochondrial oxygen consumption studies were performed in isolated heart and liver mitochondria using a XF24 extracellular flux analyser with substrates feeding electrons into complexes I, II and IV. Murine transthoracic echocardiography was conducted using a high-resolution micro ultrasound system as described previously ${ }^{45}$. Competitive transplant experiments were performed following standard protocols. Fasting glucose concentrations were determined after $12-16 \mathrm{~h}$ of fasting. For in vivo Ad-Tert/PGC-1a transduction studies, mice were transduced with $10^{9}$ virus particles per mouse and peripheral glucose levels determined 5-6 days post infection. Gluconeogenesis in isolated hepatocytes was determined following established protocols and glucose concentration is reported after protein standardization ${ }^{24}$. For p53-ER activation studies in MEFs, control or experimental cells were either treated with ethanol vehicle or 4OHT and mitochondrial mass was determined by MitoGreen and by qPCR. For promoter analysis, sequences of $2.8 \mathrm{~kb}$ (PGC-1a) and $2.6 \mathrm{~kb}$ (PGC-1 $\beta$ ), upstream of the start sites, were amplified by PCR from genomic mouse heart DNA and cloned into a luciferase reporter vector. Upstream lengths were chosen based on potential p53 binding sites as identified by TRANSFAC. For chromatin immunoprecipitation, we followed the EZ-Chip protocol (Promega) using p53 specific antibody and control IgG. Doxorubicin was administered at $7.5 \mathrm{mg}$ per kg body weight into 8-week-old mice and echocardiography was performed 7 days later. For full details, see Supplementary Methods.

\section{Supplementary Material}

Refer to Web version on PubMed Central for supplementary material.

\section{Acknowledgments}

We thank C. Bianchi, J. Moriarty, K. Marmon and E. Thompson for excellent mouse husbandry and care. We are grateful to B. Spiegelman, P. Puigserver, J. E. Dominy and J. L. Estall for providing Ad-PGC-1a and Ad-GFP virus and helpful comments on the manuscript. We thank G. I. Evan for the p53-ER construct. We appreciate input, critical comments and helpful discussions from many DePinho/Chin lab members, in particular A.-J. Chen, C. Khoo, R. Carrasco, A. Kimmelman, S. Quayle, D. Liu and R. Wiedemeyer. We acknowledge the services of the Mouse Metabolism Cores at Yale (NIH/NIDDK U24 DK-59635) and at Baylor College of Medicine (BCM) and the BCM Diabetes \& Endocrinology Research Center (DERC) grant (P30 DK079638). E.S. was supported by the Deutsche Forschungsgemeinschaft and this work and R.A.D. are supported by R01 and U01 grants from the NIH National Cancer Institute and the Robert A. and Renee E. Belfer Foundation. R.A.D. was supported by an Ellison Foundation for Medical Research Senior Scholar and an American Cancer Society Research Professor award. M.L. is a recipient of a postdoctoral fellowship from Fundación Ramón Areces.

\section{References}

1. Kirkwood TB. Understanding the odd science of aging. Cell. 2005; 120:437-447. [PubMed: 15734677]

2. Wallace DC. A mitochondrial paradigm of metabolic and degenerative diseases, aging, and cancer: a dawn for evolutionary medicine. Annu Rev Genet. 2005; 39:359-407. [PubMed: 16285865] 
3. Balaban RS, Nemoto S, Finkel T. Mitochondria, oxidants, and aging. Cell. 2005; 120:483-495. [PubMed: 15734681]

4. Guarente L. Mitochondria — a nexus for aging, calorie restriction, and sirtuins? Cell. 2008; 132:171176. [PubMed: 18243090]

5. Conley KE, Marcinek DJ, Villarin J. Mitochondrial dysfunction and age. Curr Opin Clin Nutr Metab Care. 2007; 10:688-692. [PubMed: 18089948]

6. Trifunovic A, et al. Premature ageing in mice expressing defective mitochondrial DNA polymerase. Nature. 2004; 429:417-423. [PubMed: 15164064]

7. Finley LW, Haigis MC. The coordination of nuclear and mitochondrial communication during aging and calorie restriction. Ageing Res Rev. 2009; 8:173-188. [PubMed: 19491041]

8. Arnold AS, Egger A, Handschin C. PGC-1a and myokines in the aging muscle-a mini-review. Gerontology. 2011; 57:37-43. [PubMed: 20134150]

9. Lin J, Handschin C, Spiegelman BM. Metabolic control through the PGC-1 family of transcription coactivators. Cell Metab. 2005; 1:361-370. [PubMed: 16054085]

10. Sahin E, Depinho RA. Linking functional decline of telomeres, mitochondria and stem cells during ageing. Nature. 2010; 464:520-528. [PubMed: 20336134]

11. Maser RS, DePinho RA. Connecting chromosomes, crisis, and cancer. Science. 2002; 297:565569. [PubMed: 12142527]

12. Hastie ND, et al. Telomere reduction in human colorectal carcinoma and with ageing. Nature. 1990; 346:866-868. [PubMed: 2392154]

13. Lee H-W, et al. Essential role of mouse telomerase in highly proliferative organs. Nature. 1998; 392:569-574. [PubMed: 9560153]

14. Blasco MA, et al. Telomere shortening and tumor formation by mouse cells lacking telomerase RNA. Cell. 1997; 91:25-34. [PubMed: 9335332]

15. Chin L, et al. p53 deficiency rescues the adverse effects of telomere loss and cooperates with telomere dysfunction to accelerate carcinogenesis. Cell. 1999; 97:527-538. [PubMed: 10338216]

16. Wong KK, et al. Telomere dysfunction and Atm deficiency compromises organ homeostasis and accelerates ageing. Nature. 2003; 421:643-648. [PubMed: 12540856]

17. Maier B, et al. Modulation of mammalian life span by the short isoform of p53. Genes Dev. 2004; 18:306-319. [PubMed: 14871929]

18. Tyner SD, et al. p53 mutant mice that display early ageing-associated phenotypes. Nature. 2002; 415:45-53. [PubMed: 11780111]

19. Maida Y, et al. An RNA-dependent RNA polymerase formed by TERT and the RMRPRNA. Nature. 2009; 461:230-235. [PubMed: 19701182]

20. Sarin KY, et al. Conditional telomerase induction causes proliferation of hair follicle stem cells. Nature. 2005; 436:1048-1052. [PubMed: 16107853]

21. Farazi PA, Glickman J, Horner J, Depinho RA. Cooperative interactions of p53 mutation, telomere dysfunction, and chronic liver damage in hepatocellular carcinoma progression. Cancer Res. 2006; 66:4766-4773. [PubMed: 16651430]

22. Erdmann N, Liu Y, Harrington L. Distinct dosage requirements for the maintenance of long and short telomeres in mTert heterozygous mice. Proc Natl Acad Sci USA. 2004; 101:6080-6085. [PubMed: 15079066]

23. Arany $Z$, et al. Transverse aortic constriction leads to accelerated heart failure in mice lacking PPAR- $\gamma$ coactivator 1a. Proc Natl Acad Sci USA. 2006; 103:10086-10091. [PubMed: 16775082]

24. Yoon JC, et al. Control of hepatic gluconeogenesis through the transcriptional coactivator PGC-1. Nature. 2001; 413:131-138. [PubMed: 11557972]

25. Lai L, et al. Transcriptional coactivators PGC-1a and PGC-1 $\beta$ control overlapping programs required for perinatal maturation of the heart. Genes Dev. 2008; 22:1948-1961. [PubMed: 18628400]

26. Leri A, et al. Ablation of telomerase and telomere loss leads to cardiac dilatation and heart failure associated with p53 upregulation. EMBO J. 2003; 22:131-139. [PubMed: 12505991]

27. Pannicke U, et al. Reticular dysgenesis (aleukocytosis) is caused by mutations in the gene encoding mitochondrial adenylate kinase 2. Nature Genet. 2008; 41:101-105. [PubMed: 19043417] 
28. Sankaran VG, Orkin SH, Walkley CR. $R b$ intrinsically promotes erythropoiesis by coupling cell cycle exit with mitochondrial biogenesis. Genes Dev. 2008; 22:463-475. [PubMed: 18258751]

29. Liu J, et al. Bmi1 regulates mitochondrial function and the DNA damage response pathway. Nature. 2009; 459:387-392. [PubMed: 19404261]

30. Bae BI, et al. p53 mediates cellular dysfunction and behavioral abnormalities in Huntington's disease. Neuron. 2005; 47:29-41. [PubMed: 15996546]

31. Miyagawa K, et al. Attenuation of doxorubicin-induced cardiomyopathy by endothelin-converting enzyme-1 ablation through prevention of mitochondrial biogenesis impairment. Hypertension. 2010; 55:738-746. [PubMed: 20101000]

32. Uldry M, et al. Complementary action of the PGC-1 coactivators in mitochondrial biogenesis and brown fat differentiation. Cell Metab. 2006; 3:333-341. [PubMed: 16679291]

33. Gardner JP, et al. Rise in insulin resistance is associated with escalated telomere attrition. Circulation. 2005; 111:2171-2177. [PubMed: 15851602]

34. Salpea KD, Humphries SE. Telomere length in atherosclerosis and diabetes. Atherosclerosis. 2010; 209:35-38. [PubMed: 20080237]

35. Rudolph KL, et al. Longevity, stress response, and cancer in aging telomerase-deficient mice. Cell. 1999; 96:701-712. [PubMed: 10089885]

36. Rossi DJ, et al. Deficiencies in DNA damage repair limit the function of haematopoietic stem cells with age. Nature. 2007; 447:725-729. [PubMed: 17554309]

37. Liu L, Trimarchi JR, Smith PJ, Keefe DL. Mitochondrial dysfunction leads to telomere attrition and genomic instability. Aging Cell. 2002; 1:40-46. [PubMed: 12882352]

38. Passos JF, von Zglinicki T. Mitochondria, telomeres and cell senescence. Exp Gerontol. 2005; 40:466-472. [PubMed: 15963673]

39. Veatch JR, McMurray MA, Nelson ZW, Gottschling DE. Mitochondrial dysfunction leads to nuclear genome instability via an iron-sulfur cluster defect. Cell. 2009; 137:1247-1258. [PubMed: 19563757]

40. Matoba S, et al. p53 regulates mitochondrial respiration. Science. 2006; 312:1650-1653. [PubMed: 16728594]

41. Rodgers JT, et al. Nutrient control of glucose homeostasis through a complex of PGC-1a and SIRT1. Nature. 2005; 434:113-118. [PubMed: 15744310]

42. Chen WY, et al. Tumor suppressor HIC1 directly regulates SIRT1 to modulate p53-dependent DNA-damage responses. Cell. 2005; 123:437-448. [PubMed: 16269335]

43. Cheng HL, et al. Developmental defects and p53 hyperacetylation in Sir2 homolog (SIRT1)deficient mice. Proc Natl Acad Sci USA. 2003; 100:10794-10799. [PubMed: 12960381]

44. Farazi PA, et al. Differential impact of telomere dysfunction on initiation and progression of hepatocellular carcinoma. Cancer Res. 2003; 63:5021-5027. [PubMed: 12941829]

45. Minamishima YA, et al. Somatic inactivation of the PHD2 prolyl hydroxylase causes polycythemia and congestive heart failure. Blood. 2008; 111:3236-3244. [PubMed: 18096761] 

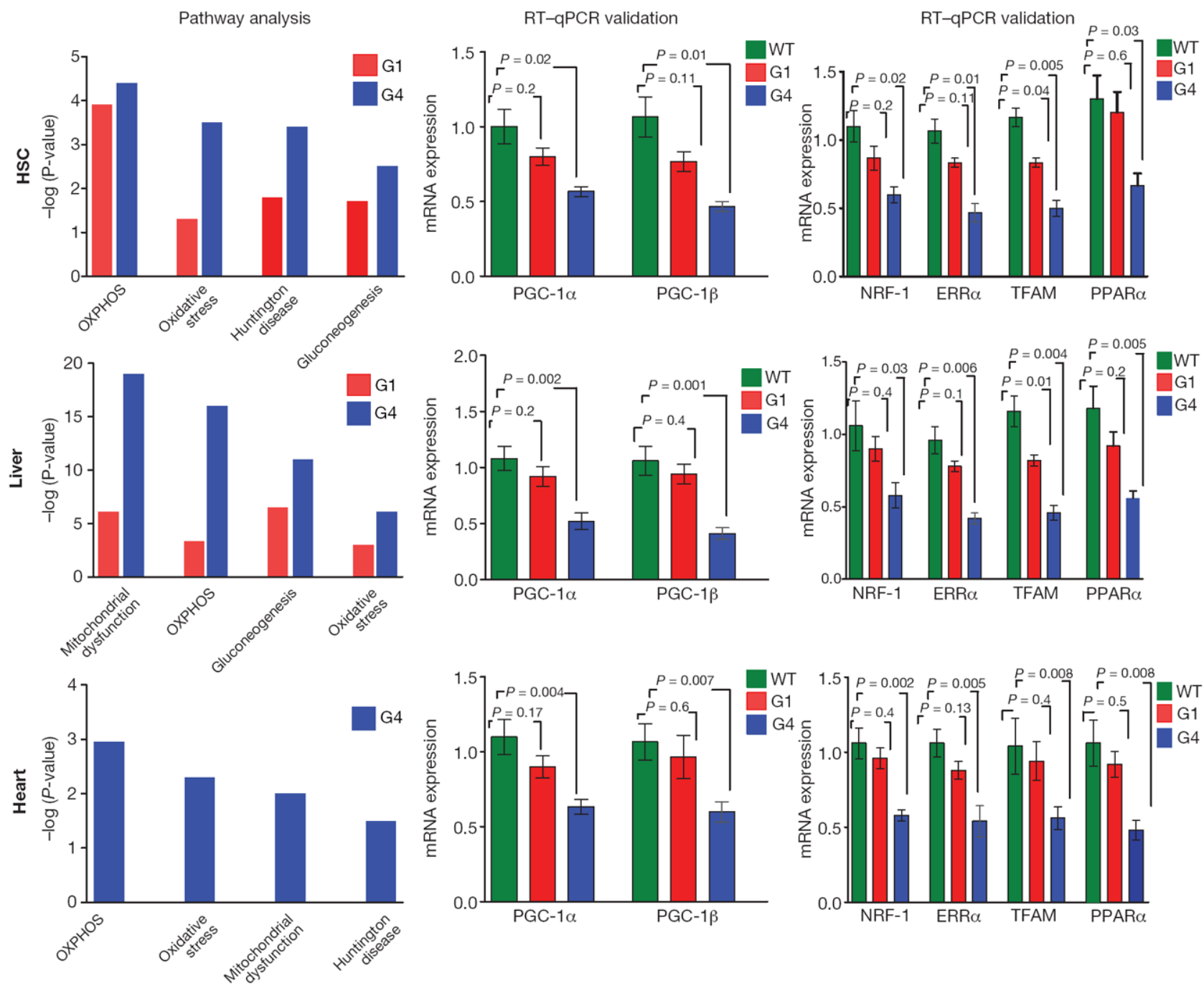

Figure 1. PGC-regulated genes and networks are repressed in telomere dysfunctional tissues Microarray-based Ingenuity pathway analysis (IPA, left) and RT-qPCR validation of PGC-1 $\alpha$, PGC-1 $\beta$ (middle) and transcriptional targets NRF-1, ERR $\alpha$, TFAM and PPARa (right) in haematopoietic stem cells (HSC), liver and heart tissues show repression of genes in the PGC network including oxidative phosphorylation (OXPHOS), mitochondrial dysfunction, gluconeogenesis, oxidative stress and Huntington Disease (HD). IPA results are expressed as $-\log$ ( $p$-value). $\Delta \Delta C$ method was used to analyse RT-qPCR data ( $n=5-8$ per group), $t$-test was used to calculate the statistical significance and error bars indicate s.e.m. 
a

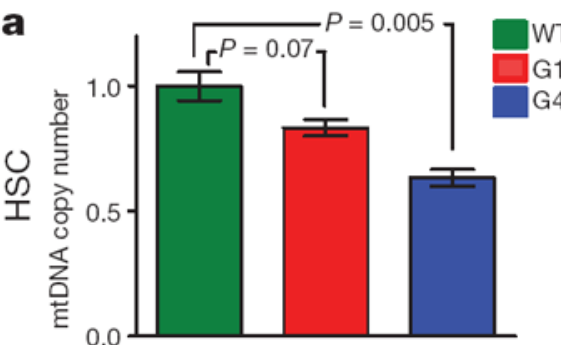

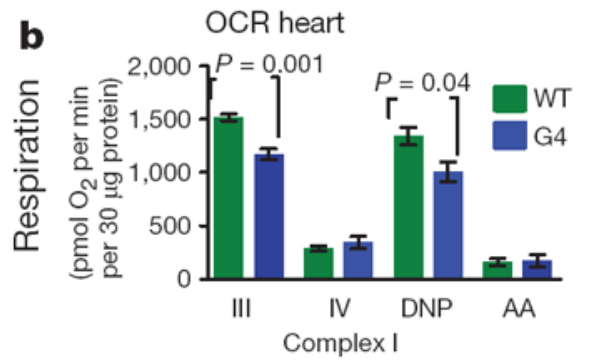

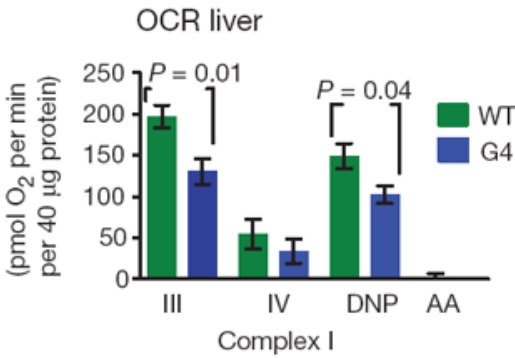

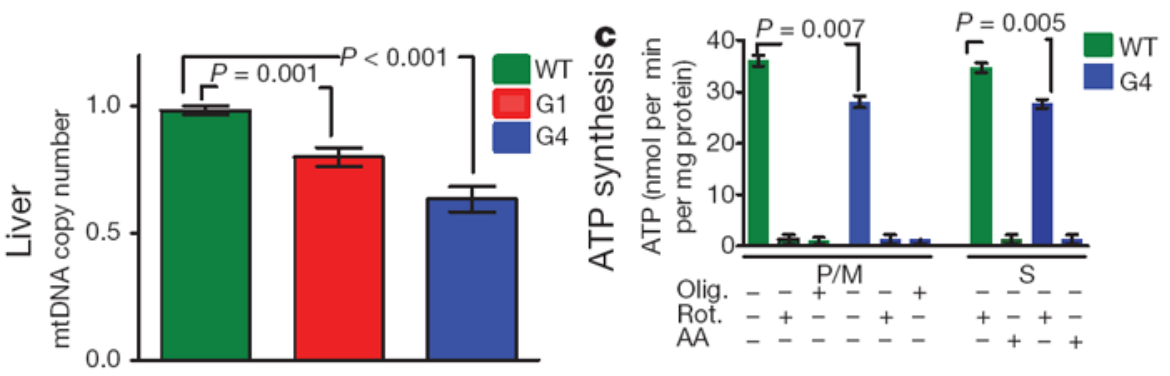
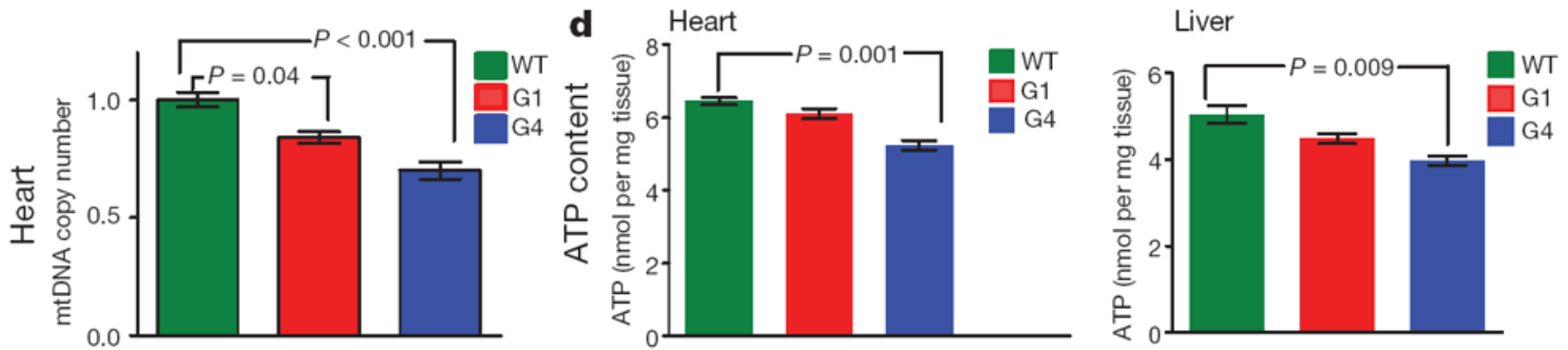

Figure 2. Telomere dysfunction is associated with reduced mitochondrial DNA content in HSC, liver and heart and impaired mitochondrial function

a, Mitochondrial DNA copy number in HSC, liver and heart $(n=5-8)$. b, Oxygen consumption rates (OCR) in heart and liver mitochondria in the presence of glutamate/ malate (liver, $n=3-5$ ) or pyruvate/malate (heart, $n=3-5$ ). State III was induced by injection of ADP. State IV was induced by inhibition of the ATP synthase with oligomycin and uncoupled respiration rates were determined by injection of 2,4-dinitrophenol (DNP). Antimycin A (AA) was used to determine background, non-mitochondrial OXPHOS, OCR. c, ATP synthesis rates in isolated heart mitochondria driven by complex I (pyruvate /malate $(\mathrm{P} / \mathrm{M})$ ) and complex II (succinate, $\mathrm{S}$ ) respiration. The specificity of the measurements is verified by the effect of inhibitors (oligomycin (Olig.), rotenone (Rot.) and antimycin A (AA) ( $n=3$ per group, duplicate measurements per sample). Succinate-dependent ATP synthesis was determined in the presence of the complex I inhibitor rotenone. d, ATP content in liver and heart tissues was determined by HPLC $(n=5)$. $t$-test was used to calculate the statistical significance and error bars indicate s.e.m. 
a
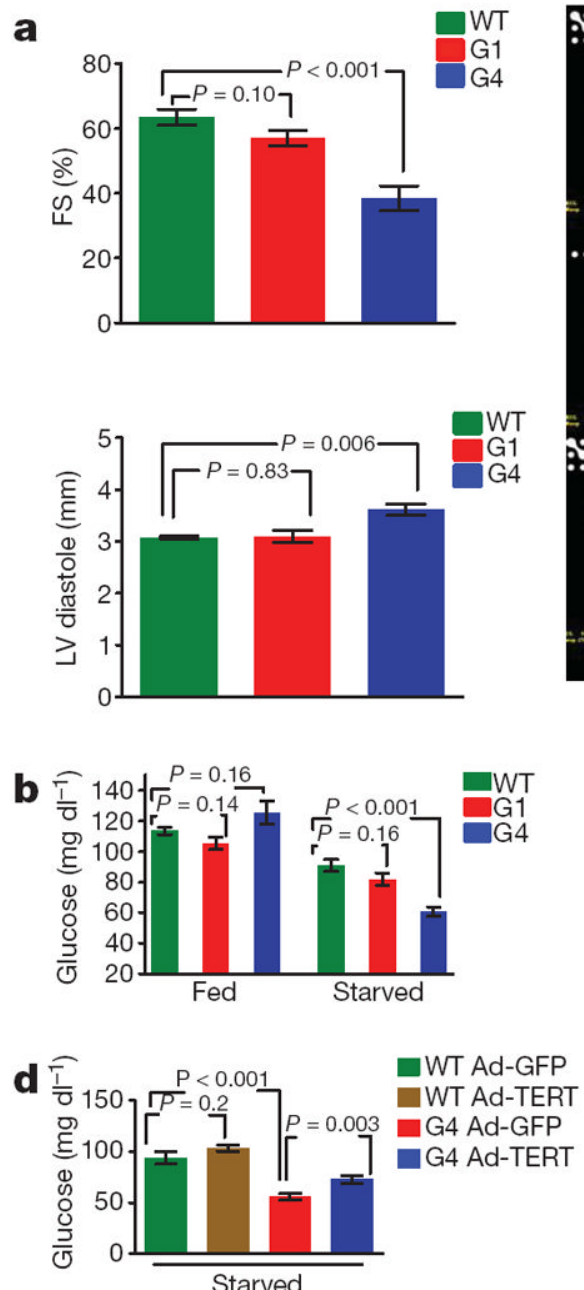

$\mathbf{f}$

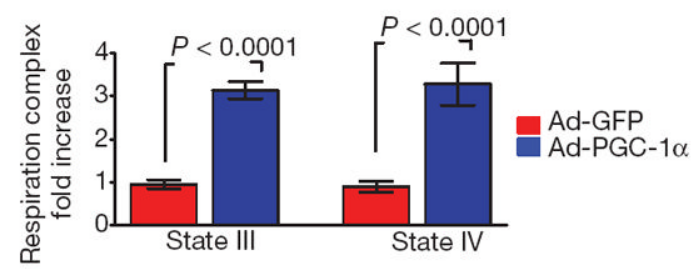

c

e
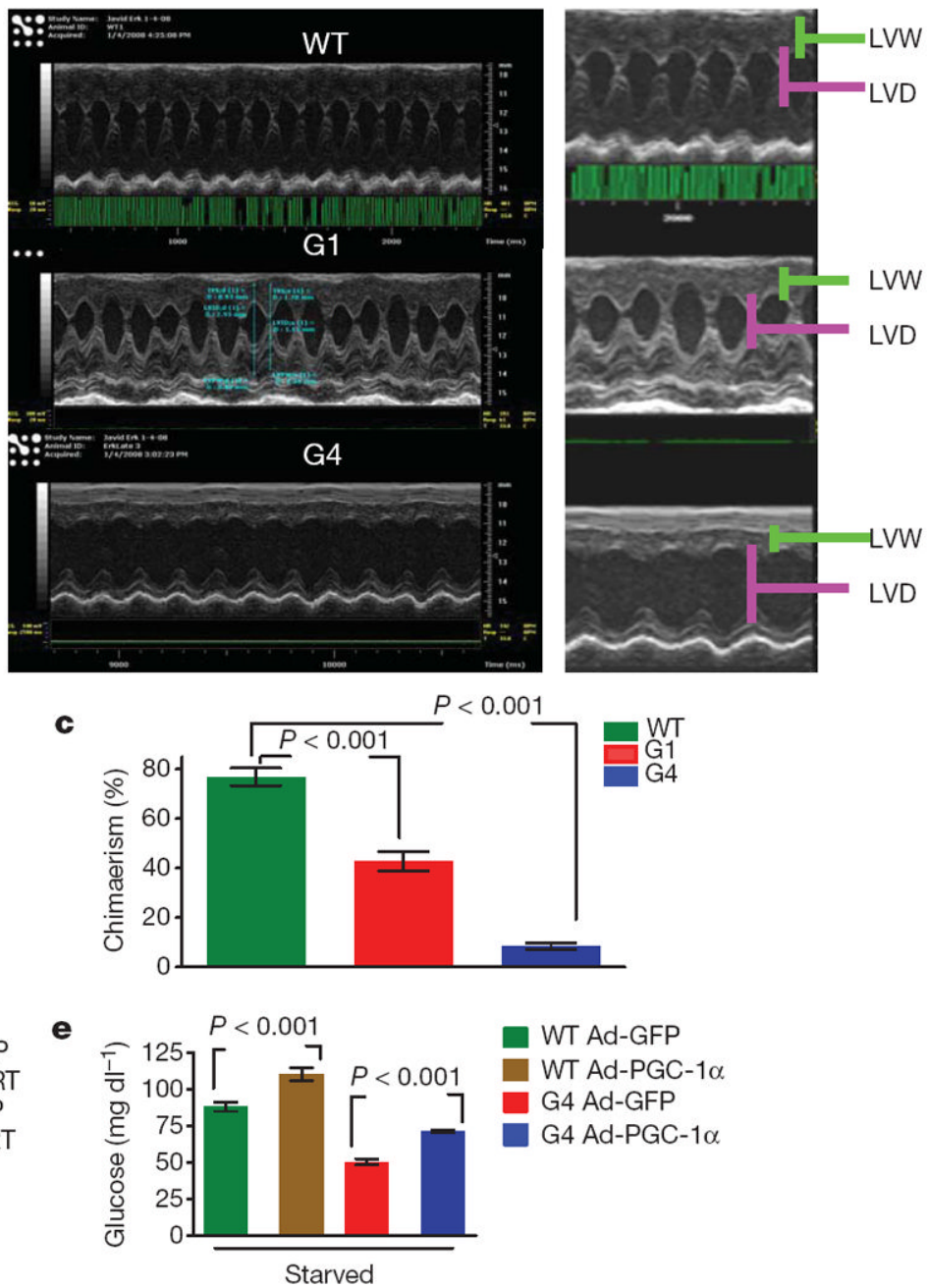

Figure 3. Telomere dysfunction induces cardiomyopathy, defective gluconeogenesis and reduced HSC reconstitution capacity

Telomerase and PGC-1a overexpression improves gluconeogenesis and mitochondrial respiration in G4 mice. a, Decreased fractional shortening (FS \%) in 15-monthold G4 mice and signs of end-stage cardiomyopathy (left ventricular diameter (LVD) increase and thinning of left ventricular wall (LVW), $n=5-8$ per genotype). $\mathbf{b}$, Glucose levels in mice under fed and starved conditions ( $n=10$ per genotype). c, Long-term repopulation capacity in competitive transplants was determined using CD45.1- and CD45.2-specific antibodies. Shown is the percentage contribution of donors after 4 months. ( $n=8$ donors per group, three recipients per donor). d, Overexpression of Tert by adenovirus attenuates gluconeogenesis defect in G4 mice ( $n=8$ per group). e, Overexpression of PGC-1a attenuates gluconeogenesis defect in G4 mice. ( $n=8-10$ per group). f, PGC-1a overexpression rescues respiration defect (complex I) in G4 liver mitochondria $(n=5$ per 
group). Student $t$-test was used to calculate the statistical differences in all assays described and error bars represent s.e.m. 
a
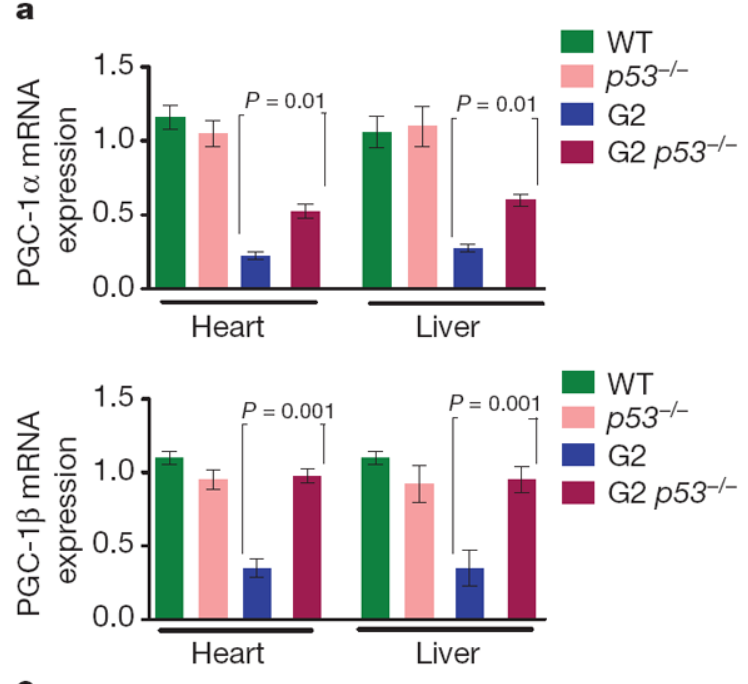

c

$$
\begin{array}{r}
1,500,000 \\
1,000,000 \\
500,000
\end{array}
$$

\begin{tabular}{ll}
$\sqsupset$ & 100,000 \\
\hdashline & 80,000
\end{tabular}

60,000

40,000

20,000
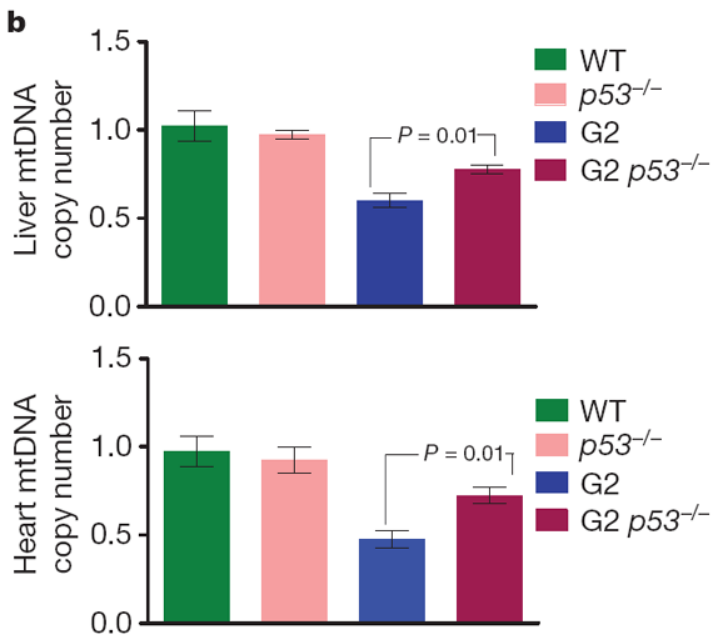
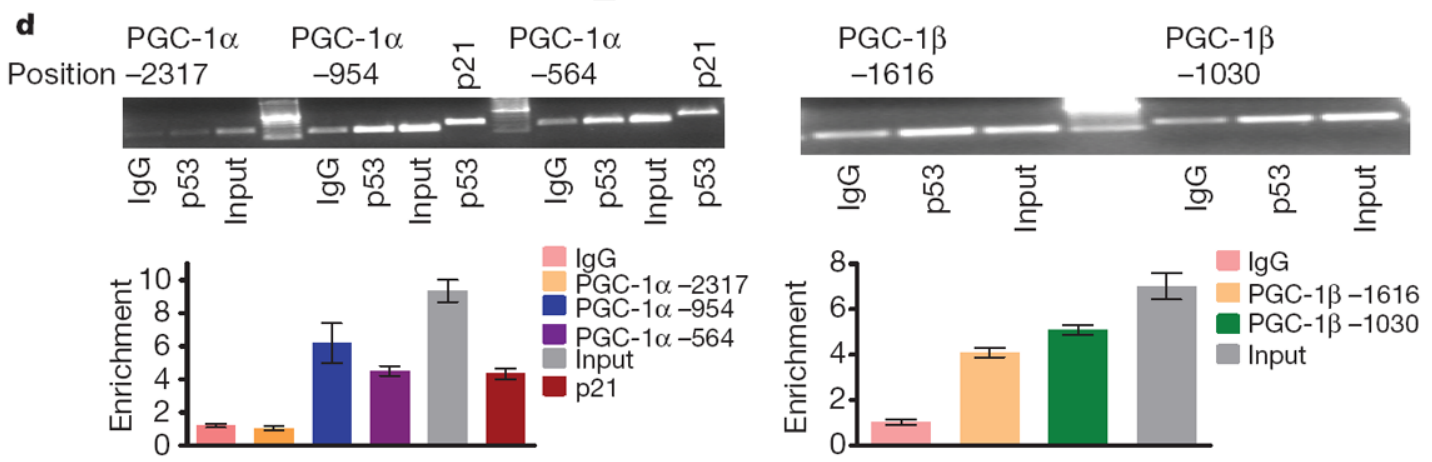

Figure 4. p53 deficiency partially rescues the transcriptional regulation of PGC-1a/ $\beta$ and mitochondrial DNA copy number

a, PGC-1 $\mathbf{a}$ and PGC-1 $\beta$ expression in liver and heart $(n=4)$. b, mtDNA quantification in liver and heart $(n=4)$. c, p53 represses PGC-1a and PGC-1 $\beta$ promoter reporters. G2 Terc $p 53^{+/+}$and G2 Terc $p 53^{-/-}$MEFs were transfected with empty reporter pGL4 or PGL4 containing various reporter fragments $(-2.8$ and $-2.6 \mathrm{~kb}$ fragment of mouse PGC-1a and PGC-1 $\beta$ ). Controls included the PG13-luc plasmid (containing 13 copies of a synthetic p53 DNA binding site) and MG15-luc (containing 15 copies of a mutated p53 DNA binding site). Shown is the average luciferase value (relative light units, RLU) of three different experiments. d, Chromatin immunoprecipitation (ChIP) showing p53 binding on the promoters of PGC-1 $\alpha$ and PGC-1 $\beta$ at indicated sites and at $\mathrm{p} 21$ site (positive control). Graphs below show quantitative results in the proximal promoter regions by qPCR (three 
independent experiments). $\Delta \Delta C$ method was used to analyse RT-qPCR data and $t$-test was used to calculate the statistical significance, error bars represent s.e.m. 
a
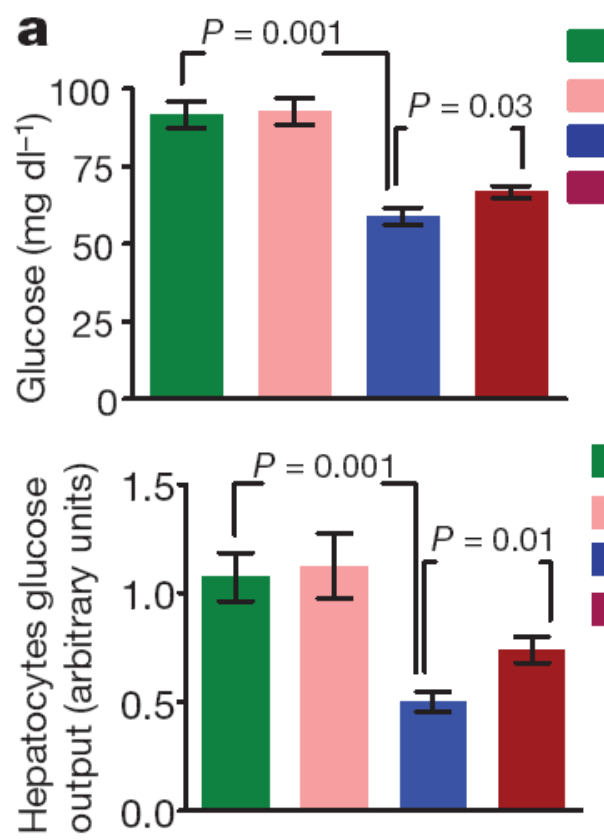

b

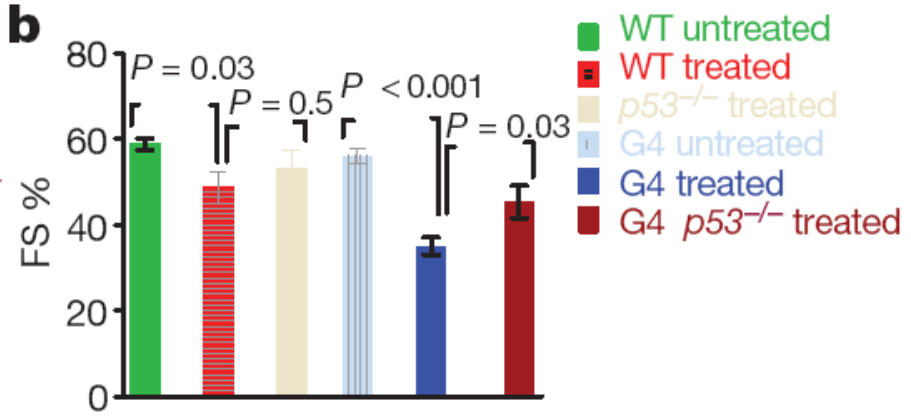

WT<smiles>C1CC[Te]C1</smiles>

G4

G4 $p 53^{-/-}$

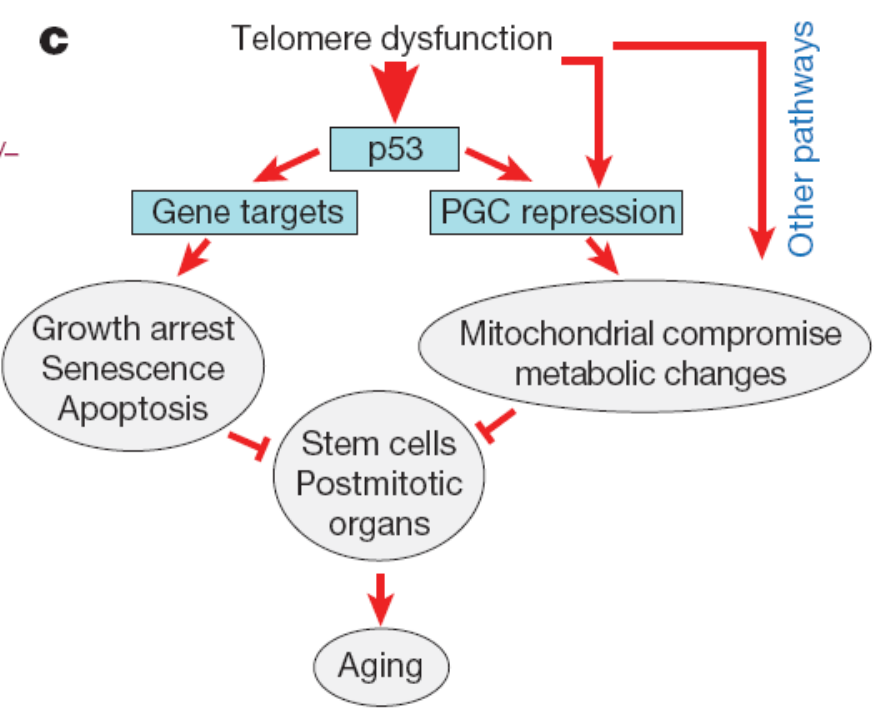

Figure 5. p53 deficiency rescues gluconeogenesis and doxorubicin-induced cardiomyopathy a, Glucose levels in mice (top) and in mediumof hepatocytes (bottom, $n=5-8$ ). $\mathbf{b}$, Doxorubicin-induced cardiomyopathy (decreased fractional shortening, FS) in G4 mice can be partially rescued by p53 deficiency ( $n=3-5, t$-test, error bars represent s.e.m.). c, Proposed model: telomere-dysfunction-induced p53 represses PGCs and induces metabolic and mitochondrial compromise. Other pathways engaged by telomere dysfunction are also involved in mediating PGC repression and mitochondrial dysfunction. Together with classical cellular outcomes of p53 activation (senescence, apoptosis and growth arrest), metabolic and mitochondrial compromise might contribute to functional organ decline in the aged. 